\title{
Comparison of intelligent charging algorithms for electric vehicles to reduce peak load and demand variability in a distribution grid
}

\author{
Kevin Mets, Reinhilde D'hulst, Chris Develder
}

\begin{abstract}
A potential breakthrough of the electrification of the vehicle fleet will incur a steep rise in the load on the electrical power grid. To avoid huge grid investments, coordinated charging of those vehicles is a must. In this paper we assess algorithms to schedule charging of plug-in (hybrid) electric vehicles as to minimize the additional peak load they might cause. We first introduce two approaches, one based on a classical optimization approach using quadratic programming, and a second one, market based coordination, which is a multi-agent system that uses bidding on a virtual market to reach an equilibrium price that matches demand and supply. We benchmark these two methods against each other, as well as to a baseline scenario of uncontrolled charging. Our simulation results covering a residential area with 63 households show that controlled charging reduces peak load, load variability, and deviations from the nominal grid voltage.
\end{abstract}

Index Terms: Smart Grid, Demand Side Management, Smart Charging, Plug-in (Hybrid) Electric Vehicles.

\section{Introduction}

Electric vehicles (EV) and plug-in hybrid electric vehicles (PHEV) are expected to gain in popularity the following years. Research estimates the number of hybrid electric vehicles in Belgium to reach $30 \%$ by 2030 [1]. This evolution is mostly driven by environmental benefits such as lowered emissions and improved fuel efficiency. However, as the electrification of the vehicle fleet is gaining momentum, it will also have an impact on the generation, transmission and distribution levels of the power grid.

Additional generation will be required to recharge the batteries of these vehicles as this requires large amounts of electrical energy which results in additional load on the power grid. However the energy required to charge these vehicles is estimated to be only $5 \%$ of total consumption in Belgium [2] in 2030. The impact on the generation and transmission levels of the power grid are therefore considered manageable on a short to medium term. However, the impact on the (residential) distribution network can be substantial, especially for high penetration levels of EVs: a single EV is estimated to double average household load during charging [3] (120V/15A 1.4kW Level 1 charger, and average residential load Southern California).

Charging electric vehicles can lead to large peak loads. Equipment installed in the power grid can be overloaded as a

K. Mets and C. Develder are with the Dept. of Information Technology IBCN at Ghent University - IBBT, G. Crommenlaan 8 Block C0 Bus 201, 9050 Ghent, Belgium, e-mail: kevin.mets@intec.ugent.be

R. D'hulst is with VITO, Boeretang 200, 2400 Mol, Belgium, e-mail: reinhilde.dhulst@vito.be result. Maintaining the power quality (e.g., voltage, unbalance, etc.) is also important to assure the correct operation of the power grid. Therefore, it is important to control and coordinate the charging of electric and plug-in hybrid electric vehicles.

The main concern of vehicle owners is to have the batteries charged by the time they need their vehicle. A certain degree of flexibility is available, because vehicles are often parked for periods of time that are longer than the time required to charge their batteries, for example during the night. We can exploit this flexibility and shift consumption to times of lower demand. This presents opportunities for the development of intelligent charging algorithms that utilize this flexibility to avoid issues in the distribution grid. These algorithms will decide on when to charge what vehicle, and potentially at what charging rate (if this can be tuned), as to achieve a certain objective (e.g., peak shaving, maximally use available green energy).

Such approaches to control and coordinate the charging of electric vehicles, that for example reduce peak load or balance demand and supply from renewable energy sources, are part of a broader context called demand side management (DSM) or demand response (DR). Instead of adapting power generation to power demand, power demand is adapted to support the optimal operation of the power grid. The application of DSM or DR is not limited to controlling the charging of electric vehicles, but also targets other residential, commercial, or industrial devices. Different approaches are considered in literature. In this work, we focus on approaches that are based on mathematical optimization and multi-agent systems. A mathematical optimization approach based on quadratic programming is presented in [4]. The aim is to minimize energy losses, and maximize the grid load factor. In earlier work [5, 6], we also explored approaches based on quadratic programming, that reduce peak load and load variability. An example of a multi-agent system is PowerMatcher [7], which is based on virtual markets, where agents bid on an electronic market to determine an equilibrium price matching demand and supply. Distributed algorithms based on dual decomposition are proposed in [8] and [9]. Other approaches are based on game theory to perform demand side management [10]. Control schemes for charging electric vehicles based on queuing theory are proposed in [11] and [12]. Clearly, there is much interest in DSM or DR algorithms, and a wide variety of methods has been proposed, to improve the operation of the distribution grid by controlling and coordinating the charging of electric vehicles (or other electrical loads).

Yet, often the proposed coordination mechanism is only benchmarked against a "business-as-usual" scenario without coordination. In this paper, we present a quadratic programming 
based coordinated charging algorithm that can serve as optimal control benchmark. We will demonstrate its usefulness in comparing it with a realistically deployable price-based coordination mechanism for DSM, in casu a market-based multi-agent system (MAS).

The contributions of this paper are: (i) an extensive analysis (beyond [5,6]) of quadratic programming (QP) based assessment of attainable peak load reduction, (ii) including associated effects on power quality, and (iii) benchmarking of a fully distributed market-based multi-agent system against the optimal $\mathrm{QP}$ results.

We also note that electric vehicles could also be used to provide ancillary services to the power grid [13], a concept known as vehicle-to-grid (V2G). An example of V2G services is storage of renewable energy. Solar and wind energy is intermittent and often the availability thereof does not coincide with the demand for energy. Electric vehicles can be charged at these moments and help balance supply and demand. The energy stored in the EVs' batteries obviously can be used later for transportation, but it could also be delivered back to the grid while the EV is still stationed at the charging point. Although this is a promising concept, we will not consider it in this work. However, both approaches we consider, can be adapted to V2G services [6].

The remainder of this paper is structured as follows: our problem statement is summarized in Section II. We discuss the algorithms considered in this paper in Section III. The case study used to evaluate the different algorithms is presented in Section IV and results are discussed in Section V. Finally, conclusions are synthesized in Section VI.

\section{Problem statement}

Charging algorithms that determine optimized charging schedules can reduce the negative effects that the additional load has on the distribution grid, and also optimize the consumption of renewable and intermittent energy sources. This paper discusses two approaches used to determine charging schedules of electric vehicles. The first approach adopts quadratic programming $(Q P)$, whereas the second approach is based on multi-agent systems (MAS) and electronic markets. The goal of both approaches is to minimize the peak load and load profile variability of the transformer load profile resulting from charging electric vehicles. This is achieved by shifting the charger loads in time and controlling the rate of charging.

The two approaches have a fundamental difference in their design. We use the QP approach in an offline setting, where we assume all events (cars arriving, departing, evolution of base load of other electrical consumers) are known in advance: the QP solution hence will result in an optimal answer to the EV charging scheduling problem. (Note that some online approaches can be straightforwardly be derived, which would lead to sub-optimal results, but these are not further discussed in this paper.) The second approach, MAS, will reflect the more realistic online situation, where we do not know beforehand what car will arrive when, but rather (re)compute the charging schedule dynamically upon each arrival. The goal of this work is to measure the differences between the two approaches.

\section{Charging algorithms}

The algorithms that form the topic of this paper determine charging schedules that control the recharging of electric vehicles. Each schedule indicates when a certain vehicle can be charged and at which charging rate.

The following sections will describe the different approaches taken. Afterwards, we compare the results from each approach to a "business-as-usual" (BAU) case in which we assume that the car immediately starts charging upon arrival at the charging point, without any form of coordination, until it is fully charged. In this BAU scenario, the charging rate is not controlled, but is fixed by the car/battery properties.

\section{A. Quadratic Programming}

In the following sections we discuss three algorithms based on quadratic programming (QP): the local, iterative global, and global algorithms. The local and iterative global algorithms have been introduced in earlier work [5]. However, we here expand on this earlier work by introducing a third algorithm, and by comparing these algorithms to an algorithm based on multiagent systems and electronic markets.

Quadratic programming is a specific type of optimization problem in which a quadratic function of several variables subject to linear constraints on these variables is optimized (minimizing or maximizing). The three algorithms are similar in nature, but differ in the amount of knowledge they posses about their surroundings, i.e. regarding the power consumption of other households and vehicles.

\section{A.1 Model parameters}

We first discuss the parameters that are present in the different quadratic programming models. The models consists of $K$ households, identified individually by the variable $k$. The simulated period of time (e.g. 24 hours) is divided in $T$ discrete time slots (e.g. 5 minutes) which are identified by the variable $t$.

We assume that the load resulting from the usage of electric appliances in each household is uncontrollable; we call this load the uncontrollable load. Each household $k$ has a load profile for the uncontrollable loads $B_{k}(t)$ that indicates the average uncontrollable load (stemming from household appliances etc.) during each time slot $t$. The aggregated energy demand of each household is limited to $L_{\max }$ (representing the grid connection capacity), expressed in Watt.

Charging electric vehicles will result in an additional load in the households. This load however is flexible as it can be shifted in time and therefore it is not part of the uncontrollable load of a household. Each vehicle has an arrival and departure time slot, respectively $\alpha_{k}$ and $\beta_{k} . B C_{k}$ indicates the maximal capacity of the battery, expressed in Wh. $C_{k}$ indicates the energy contained in the battery pack upon arrival and is also expressed in Wh. The charging rate is controllable but limited by $X_{k, \max }$.

The equations use a conversion factor, $\delta$, to calculate the energy consumed (expressed in Wh) during a certain time slot based on the load (expressed in W) during that time slot (e.g. $\delta=0.25$ assuming 15 minute time slots). 


\section{A.2 Local algorithm(QP1)}

The local (i.e. single household) scheduling method uses information about local power consumption to determine a charging schedule, i.e., we assume that the household energy consumption is known between arrival and departure time. A home energy management system could provide this information, e.g. based on historical data. The impact of other households and vehicles on the global load profile is not considered in this case. Therefore, the schedules resulting from this approach minimize local peak load and load profile variability. The quadratic programming model described below is solved for each vehicle separately upon arrival at the charging point at home.

A target load profile $T_{k}(t)$ is calculated for $t \in\left\{\alpha_{k}, \ldots, \beta_{k}\right\}$, the duration of the charging session, before determining the optimal charging schedule. The goal is for the household load profile, which includes the uncontrollable load and charger load, to approach this target profile as closely as possible. The optimal target load profile, considering the goals of minimizing the peak load and load profile variability, is formed by a constant load. The target load profile represents the constant power that should be supplied, to provision the energy requirements of the household and electric vehicle. Of course, this is not achievable, because not all devices have flexibility. The calculation of the target load at each household is defined in equation (1) and is based on the battery capacity $B C_{k}$, the current battery state $C_{k}$, the uncontrollable load $B_{k}(t)$, and the charging session duration.

$$
T_{k}(t)=\frac{\left(B C_{k}-C_{k}\right) \cdot \delta+\sum_{t^{\prime}=\alpha_{k}}^{\beta_{k}} B_{k}\left(t^{\prime}\right)}{\beta_{i}-\alpha_{i}}
$$

The following constraints apply to the optimization problem. The decision variables $X_{k}(t)$ of the optimization problem form the charging schedule and indicate the charing rate during each time slot. We define decision variables for one vehicle. The charging rate is limited by $X_{k, \max }$, and can be any as defined by constraint (2). Constraint (3) assures that the load of the household does not exceed a certain limit $L_{\max }$, e.g. set by the supplier, distribution system operator (DSO), or technical constraints (e.g. household circuitry). Finally, constraint (4) assures that the battery is fully charged after applying the charging schedule. Note that we use a very simple battery model. However, this should not significantly influence the results [14].

$$
\begin{gathered}
0 \leq X_{k}(t) \leq X_{k, \max } \\
B_{k}(t)+X_{k}(t) \leq L_{\max } \\
C_{k}+\sum_{t=\alpha_{k}}^{\beta_{k}}\left(X_{k}(t) \cdot \delta\right)=B C_{k}
\end{gathered}
$$

The objective function is defined in equation (5). A charging schedule $X_{k}(t)$ is obtained by minimizing the squared euclidean distance between the target load profile and the household load profile.

$$
\sum_{t=\alpha_{k}}^{\beta_{k}}\left(T_{k}(t)-\left(B_{k}(t)+X_{k}(t)\right)\right)^{2}
$$

\section{A.3 Iterative global algorithm (QP2)}

The iterative global algorithm also uses power consumption information, but it is not limited to local information. The algorithm is initialized by determining the load profile observed by the transformer to which the households are connected. Equation (6) is used to calculate this global load profile. The global load during each time slot $t$ is the sum of all household loads during time slot $t$.

$$
G B(t)=\sum_{k=1}^{K} B_{k}(t)
$$

The following quadratic programming model is solved separately for each vehicle that wishes to recharge its batteries. The algorithm calculates a target load profile using the global load profile instead of the local load profile as done by the local algorithms.

$$
T_{k}(t)=\frac{\left(B C_{k}-C_{k}\right) \cdot \delta+\sum_{t^{\prime}=\alpha_{k}}^{\beta_{k}} G B\left(t^{\prime}\right)}{\beta_{i}-\alpha_{i}}
$$

The constraints applied to the quadratic programming model are identical to the constraints of the local algorithm and are therefore defined in constraints (2), (3), and (4).

The objective function that is minimized to determine the charging schedule is defined by equation (8). It is based on the same principle as the local algorithm, but utilizes the global load profile instead of the local load profile. As a result, we obtain a global optimum, instead of a local optimum as is the case of the local algorithm.

$$
\sum_{t=\alpha_{k}}^{\beta_{k}}\left(T_{k}(t)-\left(G B(t)+X_{k}(t)\right)\right)^{2}
$$

After determining the charging schedule, the global load profile is updated with the load originating from the charging schedule (9), hence the iterative nature of the algorithm. As a result, future iterations will account for other households and electric vehicles that have been scheduled. This is the main difference between the local and global iterative algorithms: other households and electric vehicles that have been scheduled are accounted for when a charging schedule is determined by the iterative global algorithm.

$$
G B(t)=G B(t)+X_{k}(t), \forall t \in\left[\alpha_{k}, \beta_{k}\right]
$$

The iterative global algorithm is performed on a first-come-firstserve basis for each vehicle that arrives. However, the order in which vehicles arrive will have an impact on the charging schedule. To evaluate the impact of this order, and also to evaluate the benefits of accounting for future arrivals, we developed a third approach, which is presented in section III-A.4.

\section{A.4 Global algorithm (QP3)}

The third approach based on quadratic programming assumes knowledge about household energy consumption, and even more importantly, each future charging sessions that will occur over a certain time frame. 
A scheduling period, e.g. corresponding to a calendar day, is defined for which the charging schedules of all vehicles are determined beforehand. For each vehicle, the algorithm has to know in advance the arrival time, departure time, state-ofcharge, etc. Based on this information, charging schedules for each vehicle are determined simultaneously by solving the quadratic programming model. Note that in contrast to the local and iterative global quadratic programming model, the global model only has to be solved once to determine the charging schedule for each vehicle. The advantage of this approach is that all information is known, and therefore the flexibility is maximally used.

The global algorithm is initialized in the same way as the iterative global algorithm by calculating the the global load profile using equation (6). A set of decision variables $X_{k}(t)$ and constraints is defined for each vehicle $k$. These variables will define the charging schedule for each vehicle after minimizing the objective function (10). Again, the constraints are identical to those defined by the local algorithm in equations (2), (3), and (4).

Equation (10) illustrates that the objective function is again based on the same principle as the local and iterative global algorithm. In contrast to the local and iterative global method, the quadratic programming model now contains decision variables for each vehicle. As a result the charging schedules for each vehicle $k$ will be determined after minimizing the objective function.

$$
\sum_{t=0}^{T}\left\{T(t)-\left(G B(t)+\sum_{k=1}^{K} X_{k}(t)\right)\right\}^{2}
$$

\section{A.5 Discussion on the different QP models}

Sections III-A.2, III-A.3, and III-A.4 discuss approaches based on quadratic programming. The objective of each approach is to minimize the peak load, and reduce the variability between demand over time. Although the objective of each approach is the same (i.e. reduce the peak load), the information used to determine optimal charging schedules is different for each approach. Therefore, we can evaluate what information is needed, and has to be shared between participants, to obtain suitable results. Also, the required ICT infrastructure depends on the specific approach, as illustrated in Fig. 1.

For example, the local algorithm depends on the arrival and departure time, the energy requirements, battery charger and/or electric vehicle properties, and the predicted household energy consumption. We consider it realistic that the user provides an expected departure time (while the arrival can be detected automatically from the insertion of the plug), and battery/vehicle properties be acquired automatically (e.g. through communication with the EV). Household energy consumption information can be provided by an energy management system (e.g. the home energy box in Fig. 1), based on e.g. historical data. Therefore, all information required for the local algorithm is locally available, and assuming the household is equipped with an energy management system, the optimal charging schedule can be determined locally, and no connection to a wide-area network is required.

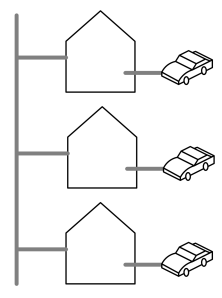

(a)

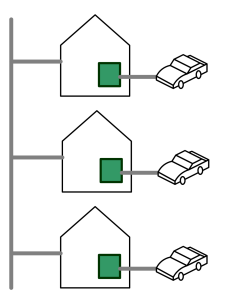

(b)

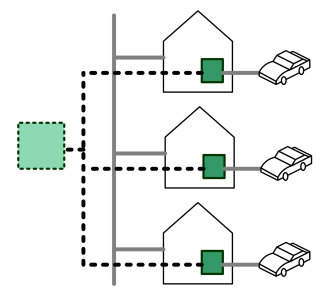

(c)
- Power line -.- Communication network

Home energy box

Global energy controller

Fig. 1. ICT infrastructure required for (a) the uncontrolled BAU case, (b) local control, (c) global/iterative control.

The iterative global and global approaches on the other hand, require information from households and vehicles to be either communicated amongst all local systems (i.e. the home energy boxes), or sent to a central controller (e.g. the Global Energy Controller in Fig. 1). Energy consumption information from all households must be aggregated, and the central controller requires information regarding arrival and departure times, energy requirements, battery and/or electric vehicle properties. Therefore, a network spanning at least the complete residential area will be required, connecting the households with the central controller. Note that privacy concerns could be raised against the global and iterative approaches, regarding the amount of information shared (since user presence and behavior could be inferred from it, e.g. through load disaggregation). We will not delve into such discussions in this paper, but rather focus on the potential technical advantages stemming from sharing that information, in terms of load shaping and power grid effects.

\section{B. Market based coordination (MAS)}

We will benchmark aforementioned (rather theoretical) QPbased approaches, with a more pragmatic coordination mechanism for EV charging coordination: a single-shot multi-unit auction market mechanism. This market based coordination mechanism also aims to prevent unwanted power peaks. The distribution grid is organized as a commodity market where agents act on behalf of the transformer and the households. An agent is a software or hardware computer system that is able to [15]:

- Make autonomous decisions

- Interact with other agents

- React, reactively and pro-actively, to changes in its environment

The commodity that is bought and sold in the market is electrical energy. In a single-shot multi-unit auction, buyers and sellers submit their bids and offers for a commodity, after which a clearing price is established to balance supply and demand $[7,16,17]$. A bidding function indicates what volume a buyer or seller is willing to trade for which price. A bidding function is constrained by the maximum volume a buyer or seller is willing or able to trade. Each buyer is allocated to consume the amount of electrical energy that he is willing to buy for the clearing price. The sellers are allocated to produce the amount of goods they are willing to sell for the clearing price. All players on the market do not know each others strategies nor bids. It should be noted that this market-based coordination approach assumes the price is only used as a control signal 


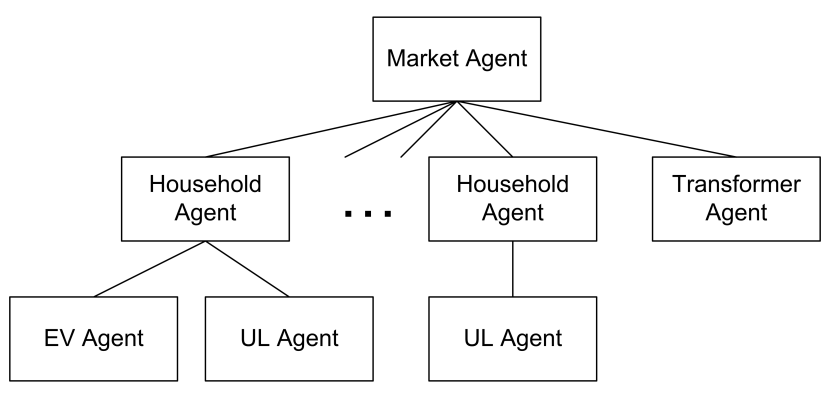

Fig. 2. Agent organization.

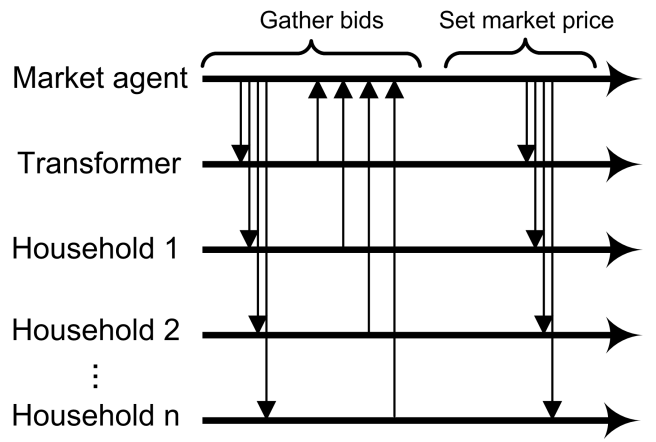

Fig. 3. Interaction between agents during one bidding round.

to stimulate devices to postpone or advance their consumption and no real-time pricing system is connected to our coordination system. The main advantage of a market based approach to coordination is that it requires no centralized planning algorithm, it scales well to a large numbers of devices as well as a large diversity of devices. Furthermore, since the only interaction between the market players is by means of bidding functions, a market based approach has less privacy issues than a centralized coordination approach.

The market-based coordination organized in the distribution grid functions as follows (see Fig. 2). Each household is represented by an agent that bids for electricity on the market. The transformer is represented by an agent as well, which acts as the sole supplier of electricity. Within a household, each device is also represented by an agent. These device agents send their bids to the household agent who aggregates these bids before sending the aggregated bid to the market. The household agents bid for an amount of electrical energy that they want to use for the next time slot and the transformer agent bids for the amount of energy it wants to deliver. In every bidding round, the market agent sends a signal to the transformer agent and the household agents, after which each agent will submit its bid. When all bids are received, the market agent aggregates the bid functions and determines the market price. This market price is communicated to the agents and based on their bids, the agents know how much energy to consume or produce. The interaction between all agents during one bidding round is depicted in Fig. 3. We assume the agents know how much their consumption will be in the next time slot when submitting a bid function.

Every household contains at least one agent representing the uncontrollable load (UL). Because the UL agent needs to be sure that the uncontrollable loads will actually get their required energy, the UL agent will always bid the maximum price for its

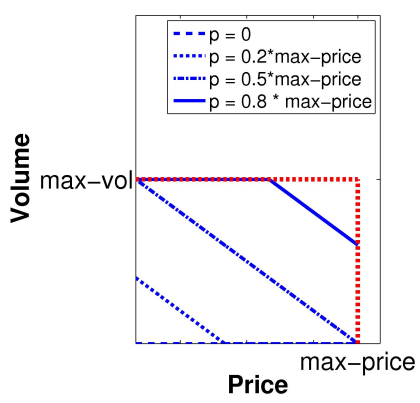

(a) EV bidding function

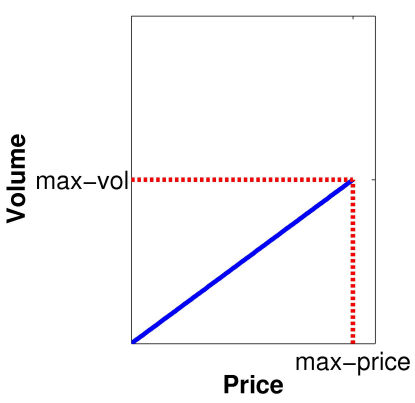

(b) transformer bidding function
Fig. 4. Bidding functions

load, as to reflect its inflexibility. The controllable device we consider in this paper will be the EV, which hence will have its separate EV agent. In this work, we assume that the EVs are able to modulate their demand, i.e., the EV chargers can demand a power between zero and the maximal power. Consequently, the bidding functions they submit are linear functions, shown in Fig. 4a. The shape of the linear bidding functions depends on the price $p$, as shown in Fig. 4a. The bidding strategy of the EV agent is to bid a price $p$ that increases linearly as the charging deadline approaches. This charging deadline is the time at which the electric vehicle has to start charging in order to be fully charged in time. An important assumption is that, in order to estimate its bid price, an EV agent is able to obtain an accurate estimation of the state-of-charge of the battery. The exact shape of the aggregated bid of a household agent thus depends on whether an EV is present or not, the bid price of that EV, the EV consumption and the consumption of the uncontrollable load.

The transformer submits a linear bid function, shown in Fig. 4b: we assume that higher costs are associated with a higher power transmitted by the transformer.

\section{Case study}

The algorithms are evaluated using three scenarios, each simulating a distribution network with a certain penetration degree of electric and plug-in hybrid electric vehicles. The different scenarios and their corresponding number of electric and plug-in hybrid electric vehicles together with the type of battery charger are defined in Table 1. We simulate a time frame of 24 hours, divided in time slots of 5 minutes.

\section{A. Power grid}

The simulated three phase distribution network is illustrated in Fig. 5, and consists of 63 households distributed over three feeders, that are connected to a distribution transformer with a rating of $250 \mathrm{kVA}$. Each household is connected to the distribution grid using a single-phase connection, which is randomly assigned to either of the three phases using a uniform distribution. The load profiles that model the power drawn by each household are based on measurements performed by VITO on a number of households in Flanders during different winter days, representing a worst case scenario, as the grid load is highest during winter in Belgium. Each house is randomly assigned one of these real-life measured load profiles which is randomly 


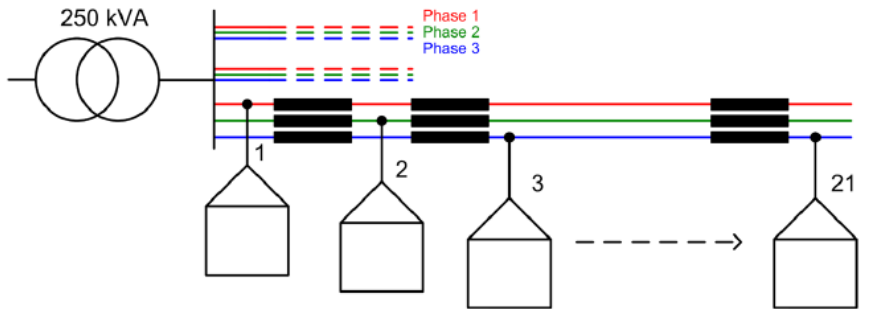

Fig. 5. Topology of the three phase distribution grid used in the simulation. It consists of 63 households, distributed over 3 feeders, and a distribution transformer with a rating of $250 \mathrm{kVA}$.

Table 1. Amount of PHEV and EV and their type of battery charger in the three different scenarios.

\begin{tabular}{|l||c|c|c|c|}
\hline Scenario & PHEV & PHEV & EV & EV \\
& $3.6 \mathrm{~kW}$ & $7.4 \mathrm{~kW}$ & $3.6 \mathrm{~kW}$ & $7.4 \mathrm{~kW}$ \\
\hline Light & 4 & 3 & 2 & 1 \\
\hline Medium & 10 & 10 & 5 & 4 \\
\hline Heavy & 17 & 16 & 7 & 7 \\
\hline
\end{tabular}

shifted in time using a uniform distribution to avoid unrealistic synchronization of loads amongst houses.

\section{B. Electric vehicles}

We assume a PHEV to have a battery capacity of $15 \mathrm{kWh}$ and an EV a battery capacity of $25 \mathrm{kWh}$. We use a linear approximation of the non-linear battery behavior. In this model, we neglect battery inefficiency and assume all power is transferred lossless through the charger into the battery. However, this should not significantly influence the results [14]. The households are provided with a single-phase connection and either a standard charger of $3.6 \mathrm{~kW}$, using $230 \mathrm{~V} 16 \mathrm{~A}$, or a fast charger of $7.4 \mathrm{~kW}$, using $230 \mathrm{~V} 32 \mathrm{~A}$. These specifications are based on the IEC 62196 standard which describes conductive charging of electric vehicles.

\section{User behavior}

It is assumed that most of the times, vehicles will be recharged at home or at work. In this paper we focus on charging at home. The plug-in times of electric vehicles are varied around 17:00 using a normal distribution with a standard deviation of 45 minutes. The charging deadline times are similarly assumed to be normally distributed around 06:00am.

\section{Results}

For each scenario (light, medium, and heavy) we selected 100 seeds to initialize the random parameters (i.e. arrival and departure times) and evaluated each algorithm for each of these 100 seeds. To compare the results from the different charging approaches, we obtained the peak load and standard deviation of each load profile and calculated the average over 100 instances for these metrics. The results presented below were obtained using our simulation environment that incorporates models of both the ICT infrastructure and the power network [18]. Fig. 6 illustrates the average transformer load profiles obtained for each scenario and algorithm. Clearly, uncontrolled charging leads to
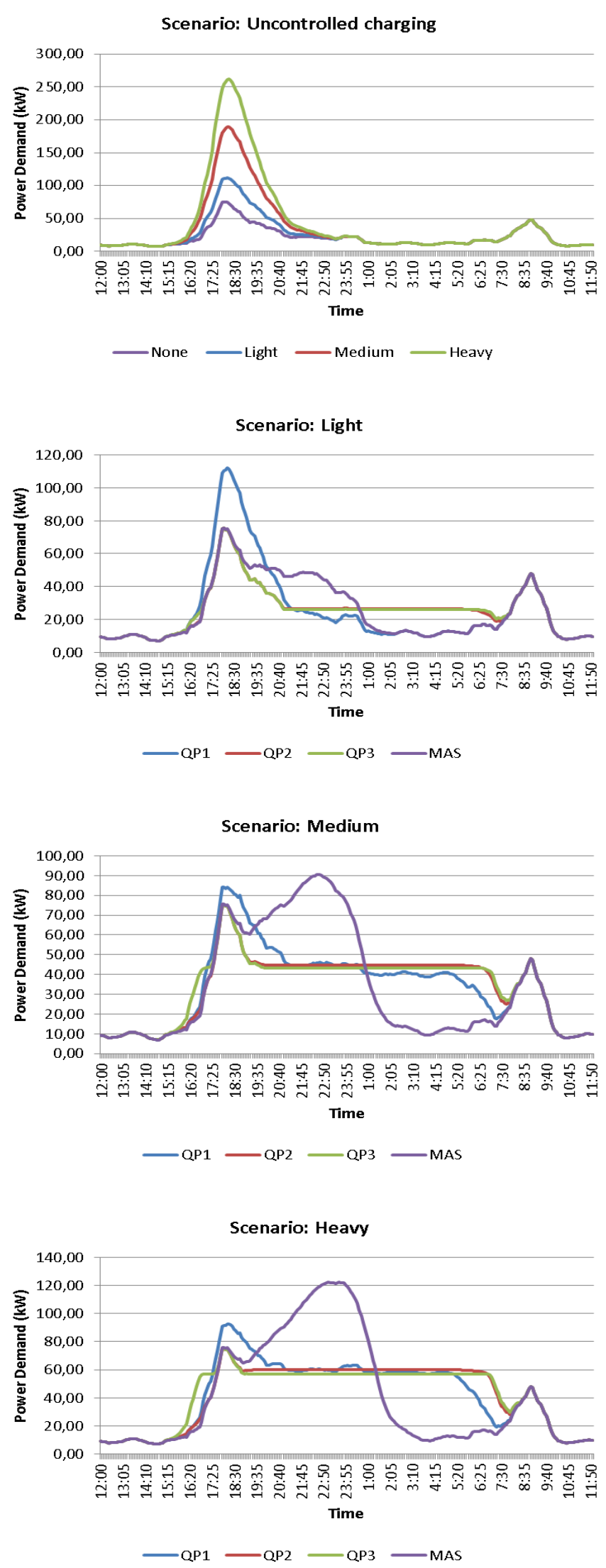

Fig. 6. Average load profiles measured at the distribution transformer. Each load profile is the average of 100 individual load profiles that were obtained for that specific scenario and algorithm. 
Table 2. Overview of the peak loads observed $(\mathrm{kW})$. The peak load is determined for each scenario and algorithm. The minimum, average, and maximum peak load are given for 100 simulations.

\begin{tabular}{|c|l|r|r|r|}
\hline Scenario & Algorithm & Minimum & \multicolumn{1}{c|}{ Mean } & Maximum \\
\hline \hline \multirow{3}{*}{ Light } & QP1 & 76.34 & 85.23 & 98.36 \\
\cline { 2 - 5 } & QP2 & 71.96 & 82.15 & 95.23 \\
\cline { 2 - 5 } & QP3 & 71.96 & 82.15 & 95.23 \\
\cline { 2 - 5 } & MAS & 71.98 & 82.28 & 95.23 \\
\hline \hline Medium & QP1 & 83.71 & 91.89 & 102.84 \\
\cline { 2 - 5 } & QP2 & 71.96 & 82.15 & 95.23 \\
\cline { 2 - 5 } & QP3 & 71.96 & 82.15 & 95.23 \\
\cline { 2 - 5 } & MAS & 86.71 & 93.19 & 99.09 \\
\hline \hline Heavy & QP1 & 90.20 & 99.23 & 110.21 \\
\cline { 2 - 5 } & QP2 & 71.96 & 82.15 & 95.23 \\
\cline { 2 - 5 } & QP3 & 71.96 & 82.15 & 95.23 \\
\cline { 2 - 5 } & MAS & 116.91 & 125.78 & 137.88 \\
\hline
\end{tabular}

a substantial increase in peak load. However, controlled charging approaches are able to reduce this peak load. A more detailed discussion is provided in the following sections.

\section{A. Total energy consumption}

Electric vehicles form an additional load on the power grid when being recharged. This additional load obviously leads to more energy consumption than the case without EVs. This is observed in the light and medium scenarios where total energy consumption rises with $22 \%$ and $63 \%$. In the heavy scenario energy consumption is doubled. Clearly, no coordination mechanism can reduce that total load increase, but rather shift the EV load in time as to minimize peak load increases. This is discussed next.

\section{B. Impact of uncontrolled charging on the peak load}

We start the discussion of the results by looking at the impact of uncontrolled charging on the peak load. Uncontrolled charging has a significant impact on the peak load because the charging coincides with the existing evening peak load. On average it leads to almost 1.5 times the peak load of current electricity consumption in a residential area if we consider the light scenario. Uncontrolled charging in the medium and heavy scenario on average leads to a peak load that is 2.4 and 3.3 times the existing peak load. The peak load does not exceed the transformer rating in the light and medium scenarios, however it exceeds the transformer rating in $88 \%$ of the simulated cases (i.e. for 88 out of 100 random seed choices).

\section{Peak load reduction by controlled charging}

As we have seen in the previous section, uncontrolled charging leads to a higher peak load, because the charging coincides with the existing evening peak load. The charging algorithms presented in this paper aim to reduce the peak load as much as possible, preferably to the same level as in the case without EVs.
Table 3. Peak load reductions. QP1 = Local, QP2 = Iterative Global, QP3 = Global, MAS = Multi Agent

\begin{tabular}{|l||c|c|c||c|}
\hline \multicolumn{1}{|c||}{} & \multicolumn{4}{c|}{ Peak Load $\searrow$} \\
\hline Scenario & QP1 & QP2 & QP3 & MAS \\
\hline \hline Light & $29.62 \%$ & $32.16 \%$ & $32.16 \%$ & $32.00 \%$ \\
\hline Medium & $53.84 \%$ & $58.73 \%$ & $58.73 \%$ & $53.19 \%$ \\
\hline Heavy & $63.76 \%$ & $70.00 \%$ & $70.00 \%$ & $54.04 \%$ \\
\hline
\end{tabular}

Table 2 and Table 3 summarize the impact on the peak load by the different energy control strategies. The energy control strategies are able to reduce the peak load of uncontrolled charging, by shifting the vehicle loads in time and controlling the rate of charging. In the light scenario, the local method (QP1) achieves a peak load reduction of $29.62 \%$ compared to the BAU scenario (i.e. uncontrolled charging), while the iterative (QP2), global (QP3) and multi-agent market (MAS) based methods all achieve a peak reduction of approximately $32 \%$. In the medium scenario, the local and multi-agent market based method achieve similar results: $53.84 \%$ and $53.19 \%$. The iterative and global methods both achieve a peak reduction of $58.73 \%$. When we consider the heavy scenario, the multi-agent market based method achieves a reduction of $54.04 \%$, the local method $63.76 \%$, and the iterative and global method both achieve a reduction of $70.00 \%$ compared to the BAU scenario. These results give an indication of what the impact is on the peak load, but we are more interested in knowing how much of the additional peak load that was the result of uncontrolled charging can be shifted.

The iterative and global methods are able to fully reduce the peak load to the original level before electric vehicles were introduced to the distribution grid. The local method however, removes only $92 \%$ of the additional peak load that is added by uncontrolled charging. The reason for this being that the local algorithm only considers peak loads in each household individually. The vehicle load is shifted in time to not coincide with the peak loads in that household. However, that local peak does not necessarily coincide with the overall peak load. The market based method is also unable to fully remove the additional peak load that is the result of charging electric vehicles: $99.64 \%$ of the additional peak load is removed in the light scenario, $90.64 \%$ in the medium scenario and $77.15 \%$ in the heavy scenario.

\section{Load profile variability}

The load profile variability is another interesting factor as it influences dispatching of generators. We measure it by calculating the standard deviation between the values of the load profile. We list the standard deviation of the transformer load over time in Table IV, and summarize its reduction compared to the BAU case in Table V. Each algorithm is able to reduce the standard deviation of the values of the load profile compared to the BAU scenario. However, there is a big difference between the methods based on quadratic programming and the market based multi-agent system. The results regarding the peak load for the iterative and global algorithm where identical, however there is a difference when considering the variance of the load profiles. The global algorithm is able to determine the most optimal so- 
Table 4. Standard deviation

\begin{tabular}{|c|l|r|r|r|}
\hline Scenario & Algorithm & Minimum & Mean & Maximum \\
\hline \hline \multirow{3}{*}{ Light } & QP1 & 15.66 & 16.17 & 16.85 \\
\cline { 2 - 5 } & QP2 & 14.18 & 14.57 & 15.24 \\
\cline { 2 - 5 } & QP3 & 14.11 & 14.49 & 15.18 \\
\cline { 2 - 5 } & MAS & 17.95 & 18.65 & 19.48 \\
\hline \hline Medium & QP1 & 18.79 & 19.80 & 20.80 \\
\cline { 2 - 5 } & QP2 & 16.56 & 17.38 & 18.19 \\
\cline { 2 - 5 } & QP3 & 15.55 & 16.78 & 17.76 \\
\cline { 2 - 5 } & MAS & 27.19 & 28.64 & 29.89 \\
\hline \hline Heavy & QP1 & 23.35 & 24.80 & 26.20 \\
\cline { 2 - 5 } & QP2 & 21.34 & 22.56 & 24.02 \\
\cline { 2 - 5 } & QP3 & 19.46 & 21.30 & 22.62 \\
\cline { 2 - 5 } & MAS & 36.66 & 38.71 & 40.57 \\
\hline
\end{tabular}

Table 5. Reduction of the standard deviation. QP1 = Local, QP2 = Iterative Global, QP3 = Global, MAS = Multi Agent

\begin{tabular}{|l||c|c|c||c|}
\hline \multicolumn{1}{|c||}{} & \multicolumn{4}{c|}{ Standard deviation $\searrow$} \\
\hline Scenario & QP1 & QP2 & QP3 & MAS \\
\hline \hline Light & $35.24 \%$ & $41.63 \%$ & $41.94 \%$ & $25.29 \%$ \\
\hline Medium & $55.01 \%$ & $60.50 \%$ & $61.88 \%$ & $34.91 \%$ \\
\hline Heavy & $60.22 \%$ & $63.82 \%$ & $65.84 \%$ & $38.80 \%$ \\
\hline
\end{tabular}

lution as it has the most information available, whereas if we only consider peak load, the iterative and global method have the same results. Note that the market based MAS system does not seem to be able to reach the flat load profile as achieved by the QP methods.

\section{E. Power quality}

The peak load and load profile variability are mainly of concern to assess production and grid capacity. Yet, as noted before, the introduction of EVs risks to cause additional problems in the distribution grid that historically was not dimensioned to cater for EVs. Using our integrated ICT- and power network simulator [18], we also assessed the impact of coordination mechanisms QP1 and QP2 on the power quality in terms of variations in voltage magnitude. As just discussed, we achieve substantial improvements in terms of peak power and demand variability reduction, using realistic assumptions on the required information. According to the EN50160 standard, voltage deviations up to $10 \%$ are acceptable in distribution grids.

First, we evaluated how often voltage deviations exceeding $10 \%$ occur during a 24 hour time period, divided in 288 time slots of 5 minutes. Table 6 gives an overview of the average number of time slots during which such deviations occur. We obtained these averages by counting the number of time slots in which deviations exceeding $10 \%$ occurred somewhere in the residential area for each experiment (using a different random seed), and calculated the average. Large $\mathrm{P}(\mathrm{H}) \mathrm{EV}$ penetration degrees lead to deviations occurring more often. For the heavy scenario, which corresponds to the worst case, uncontrolled charg-
Table 6. Average number of 15 minute time slots (out of the 288 time slots over the course of the considered one day period) during which voltage deviations exceeding $10 \%$ are observed.

\begin{tabular}{|l||c|c|c|}
\hline Scenario & BAU & QP1 & QP2 \\
\hline \hline Light & 22.17 & 3.90 & 3.31 \\
\hline Medium & 38.01 & 4.52 & 5.32 \\
\hline Heavy & 45.51 & 3.92 & 9.30 \\
\hline
\end{tabular}

Table 7. Average and maximum magnitude of voltage deviations.

\begin{tabular}{|l||c|c||c|c||c|c|}
\hline \multicolumn{1}{|c||}{} & \multicolumn{2}{c||}{ BAU } & \multicolumn{2}{c||}{ QP1 } & \multicolumn{2}{c|}{ QP2 } \\
\hline Scenario & AVG & MAX & AVG & MAX & AVG & MAX \\
\hline \hline Light & $20 \%$ & $29 \%$ & $13 \%$ & $19 \%$ & $13 \%$ & $18 \%$ \\
\hline Medium & $29 \%$ & $60 \%$ & $13 \%$ & $22 \%$ & $13 \%$ & $20 \%$ \\
\hline Heavy & $37 \%$ & $65 \%$ & $12 \%$ & $20 \%$ & $14 \%$ & $22 \%$ \\
\hline
\end{tabular}

ing on average leads to voltage deviations exceeding $10 \%$ for 45.51 time slots, or approximately $16 \%$ of the time slots. However, controlled charging reduces this number: if we consider the heavy scenario again, QP1 leads to 3.92 time slots, or approximately $1 \%$ of the time slots, and QP2 leads to 9.30 time slots, or approximately $3 \%$ of the time slots.

Next, we evaluated how large the deviations from the nominal voltage are. Results are summarized in Table 7. We only considered experiments during which at least one voltage deviation exceeding $10 \%$ occurred. For each of those experiments, we determined the maximum voltage deviation that occurred. The maximum and average values are given for each set of experiments in Table 7. Large penetration degrees of $\mathrm{P}(\mathrm{H}) \mathrm{EV}$ lead to larger voltage deviations. For the heavy scenario, the average maximum deviation observed for uncontrolled charging is $37 \%$ of the nominal voltage, and the maximum deviation observed over all experiments is $65 \%$. These deviations are much larger than the $10 \%$ required by the EN50160 standard. However, controlled charging reduces the magnitude of the deviations. The average maximum deviation for QP1 is $12 \%$ in the heavy scenario, and the maximum deviation observed over all experiments is $20 \%$. For QP2 we obtain respectively $14 \%$ and $22 \%$.

Based on the results summarized in Table 6 and Table 7, we can conclude that the QP1 approach in general results in the most optimal results. The QP1 or local approach aims to reduce the local or household peak load. Therefore, the load at each node in the grid will be as low as possible, resulting in smaller voltage deviations. The QP2 or iterative approach on the other hand, aims at reducing the transformer peak load. Individual household peak loads do not necessarily coincide with the peak load at the transformer level. Therefore, it is possible that household peak load is increased, which increases the voltage deviation.

\section{F. Discussion}

The results from the approaches based on quadratic programming are superior in terms of peak load and load profile variance reduction compared to those of the multi-agent system. How- 
ever, the results from the market based MAS system require less stringent knowledge of the load profiles, and also only exchange very limited information compared to the QP-methods. The multi-agent system has the added advantage of being a truly dynamic and flexible approach: via tweaking of the bidding curves, the optimization can be steered towards other objectives. The QP approach is more strict, and more cumbersome to adapt to different objectives. Nevertheless, the QP method is extremely useful to assess what the best possible result is, and hence serves as an optimal benchmark. In our case, it thus reveals that there is still substantial room for improving the market based MAS approach (e.g. peak reduction of $54.04 \%$ vs $70.00 \%$ for QP, variability reduction of $38.80 \%$ vs $65.84 \%$ for QP). This does not mean that the approaches based on quadratic programming are useless, as they determine the most optimal solutions and therefore can be used to benchmark other algorithms.

\section{Conclusion}

Uncontrolled charging of electric vehicles for substantial penetration would result in increases in peak load (we noted for the worst case scenario more than doubling the peak load observed in the distribution grid without electric vehicles). We presented two classes of EV charging coordination: based on classical quadratic programming $(\mathrm{QP})$ on the one hand, and marketbased multi-agent systems (MAS) on the other. The aim of both in the considered case studies is to reduce the peak load and the load variability in a distribution grid. We considered three quadratic programming approaches, assuming different knowledge of components within the grid. We provide simulation results, using a combined ICT and power simulator [18] for a residential area consisting of 63 households and different penetration degrees of electric vehicles. Peak reductions ranging from $29 \%$ up to $70 \%$ are achievable, compared to a businessas-usual scenario in which vehicles are charged without control and coordination. Variability in demand is decreased ranging from $25 \%$ up to $65 \%$. The QP method mainly serves as benchmark, since real-life deployment may be hampered by its requirement to communicate expected load profiles (e.g. based on historical measurements). The MAS approach, while requiring modest knowledge of the expected future load and imposing little communication, achieves in the range of $32.00 \%$ to $54.04 \%$ (vs $32.16 \%$ to $70.00 \%$ for QP-global) peak reduction. We conclude that future work is required to further tune and optimize e.g. MAS systems to closer achieve the optimum found by QP. We also evaluated the impact of our coordinated charging approaches in terms of power quality, under the form of voltage magnitude variations. While the objectives as formulated in our approaches do not explicitly include the voltage as a parameter to be optimized, we do note that the coordinated charging strategies reduce the observed voltage deviations (measured as differences from the nominal voltage greater than 10\%).

\section{Acknowledgment}

K. Mets would like to thank the Institute for the Promotion of Innovation by Science and Technology in Flanders (IWT) for financial support through his Ph. D. grant. C. Develder is supported in part by the Research Foundation Flanders (FWO-Vl.) as a post-doctoral fellow.

\section{REFERENCES}

[1] S. Logghe, B. V. Herbruggen, and B. V. Zeebroeck, "Emissions of Road Traffic in Belgium,” Transport \& Mobility Leuven, Tech. Rep., January 2006.

[2] K. Clement, K. V. Reusel, and J. Driesen, "The Consumption of Electrical Energy of Plug-in Hybrid Electric Vehicles in Belgium," in Proc. European Ele-Drive Conference (EET-2007), Brussels, Belgium, June 2007, p. $10 \mathrm{pp}$.

[3] A. Ipakchi and F. Albuyeh, "Grid of the future," IEEE Power and Energy Magazine, vol. 7, no. 2, pp. 52 -62, March-April 2009.

[4] K. Clement-Nyns, E. Haesen, and J. Driesen, "The Impact of Charging Plug-In Hybrid Electric Vehicles on a Residential Distribution Grid," IEEE Transactions on Power Systems, vol. 25, no. 1, pp. 371-380, February 2010.

[5] K. Mets, T. Verschueren, W. Haerick, C. Develder, and F. D. Turck, "Optimizing smart energy control strategies for plug-in hybrid electric vehicle charging," in Proc. 12th IEEE/IFIP Network Operations and Management Symposium (NOMS 2010), Osaka, Japan, 2010, pp. 293-299.

[6] K. Mets, T. Verschueren, F. De Turck, and C. Develder, "Exploiting v2g to optimize residential energy consumption with electrical vehicle (dis)charging," in Proc. 2011 IEEE 2nd Int. Conf. on Smart Grid Communications - IEEE 1st Int. Workshop on Smart Grid Modeling and Simulation (SGMS), Brussels, Belgium, October 2011, pp. $7-12$.

[7] J. K. Kok, C. J. Warmer, and I. G. Kamphuis, "PowerMatcher: Multiagent Control in the Electricity Infrastructure," in Proc. of the 4th International Joint Conference on Autonomous Agents and Multi-Agent Systems (AAMAS '05), New York, NY, USA, 2005, pp. 75-82.

[8] P. Samadi, A. Mohsenian-Rad, R. Schober, V. Wong, and J. Jatskevich, "Optimal real-time pricing algorithm based on utility maximization for smart grid," in Proc. First IEEE International Conference on Smart Grid Communications (SmartGridComm), Gaithersburg, Maryland, USA, October 2010, pp. $415-420$.

[9] N. Gatsis and G. B. Giannakis, "Residential load control: Distributed scheduling and convergence with lost ami messages," IEEE Transactions on Smart Grid, vol. 3, no. 2, pp. 770-786, 2012.

[10] A. Mohsenian-Rad, V. Wong, J. Jatskevich, R. Schober, and A. LeonGarcia, "Autonomous demand-side management based on game-theoretic energy consumption scheduling for the future smart grid," IEEE Transactions on Smart Grid, vol. 1, no. 3, pp. 320 -331, 2010.

[11] K. Turitsyn, N. Sinitsyn, S. Backhaus, and M. Chertkov, "Robust broadcast-communication control of electric vehicle charging," in Proc. First IEEE International Conference on Smart Grid Communications (SmartGridComm), Gaithersburg, Maryland, USA, 2010, pp. 203-207.

[12] M. Erol-Kantarci, J. H. Sarker, and H. T. Mouftah, "Analysis of plug-in hybrid electrical vehicle admission control in the smart grid," in Proc. IEEE 16th International Workshop on Computer Aided Modeling Analysis and Design of Communication Links and Networks (CAMAD), Kyoto, Japan, Jun. 10-11 2011, pp. 72-76.

[13] W. Kempton and J. Tomic, "Vehicle-to-grid power implementation: From stabilizing the grid to supporting large-scale renewable energy," Journal of Power Sources, vol. 144, pp. 280-294, 2005.

[14] O. Sundström and C. Binding, "Optimization methods to plan the charging of electric vehicle fleets," in Proc. of the ACEEE International Conference on Control, Communication and Power Engineering, Chennai, India, July 28 2010, pp. 323-328.

[15] J. Bradshaw, Software Agents. AAAI Press/The MIT PRess, 1997.

[16] R. Wilson, "Auctions of Shares," Quarterly Journal of Economics, vol. 93, no. 4, pp. 675-689, 1979.

[17] M. Hommelberg, C. Warmer, I. Kamphuis, J. Kok, and G. Schaeffer, "Distributed control concepts using multi-agent technology and automatic markets: an indispensable feature of smart power grids," in Proc. 2007 IEEE Power Engineering Society General Meeting, Tampa, Florida, USA, 2007, p. 7 pp.

[18] K. Mets, T. Verschueren, C. Develder, T. L. Vandoor, and L. Vandevelde, "Integrated simulation of power and communication networks for smart grid applications," in Proc. IEEE 16th International Workshop on Computer Aided Modeling Analysis and Design of Communication Links and Networks (CAMAD), Kyoto, Japan, 10-11 June 2011, pp. 62-66. 